\title{
Chirped Peregrine solitons in a class of cubic-quintic nonlinear Schrödinger equations
}

\author{
Shihua Chen,,${ }^{1,2, *}$ Fabio Baronio, ${ }^{3}$ Jose M. Soto-Crespo, ${ }^{4, \dagger}$ Yi Liu, ${ }^{2}$ and Philippe Grelu ${ }^{5, \ddagger}$ \\ ${ }^{1}$ Department of Physics, Southeast University, Nanjing 211189, China \\ ${ }^{2}$ Laboratoire d'Optique Appliquée, ENSTA ParisTech, CNRS, Ecole Polytechnique, \\ 828 Boulevard des Maréchaux, 91762 Palaiseau Cedex, France \\ ${ }^{3}$ INO CNR and Dipartimento di Ingegneria dell'Informazione, Università di Brescia, Via Branze 38, 25123 Brescia, Italy \\ ${ }^{4}$ Instituto de Óptica, Consejo Superior de Investigaciones Científicas, Serrano 121, Madrid 28006, Spain \\ ${ }^{5}$ Laboratoire ICB, UMR No. 6303, CNRS, Université Bourgogne Franche-Comté, 9 Avenue A. Savary, F-21078 Dijon, France
}

(Received 11 April 2016; published 7 June 2016)

\begin{abstract}
We shed light on the fundamental form of the Peregrine soliton as well as on its frequency chirping property by virtue of a pertinent cubic-quintic nonlinear Schrödinger equation. An exact generic Peregrine soliton solution is obtained via a simple gauge transformation, which unifies the recently-most-studied fundamental rogue-wave species. We discover that this type of Peregrine soliton, viable for both the focusing and defocusing Kerr nonlinearities, could exhibit an extra doubly localized chirp while keeping the characteristic intensity features of the original Peregrine soliton, hence the term chirped Peregrine soliton. The existence of chirped Peregrine solitons in a self-defocusing nonlinear medium may be attributed to the presence of self-steepening effect when the latter is not balanced out by the third-order dispersion. We numerically confirm the robustness of such chirped Peregrine solitons in spite of the onset of modulation instability.
\end{abstract}

DOI: 10.1103/PhysRevE.93.062202

\section{INTRODUCTION}

Photonics has recently been considered as a fertile, reproducible, and safe ground for experimental realization of rogue waves, since the latter were first discovered within the supercontinuum generation dynamics [1]. The versatility of optical rogue-wave experiments spans from the generation of the deterministic Peregrine soliton in an optical fiber [2] to the observation of dissipative rogue waves in the vicinity of mode-locked fiber laser operation [3] and from the recording of three-dimensional (3D) extreme waves in the speckle pattern of a spatially modulated optical beam [4] to the realization of dark rogue waves in a randomly birefringent telecommunication fiber [5]. Optical rogue-wave experimental research is echoing intense theoretical activity whose purpose is to understand the fundamental origin of rogue waves while unveiling their diversity [6,7].

Among all theoretical findings, the Peregrine soliton, which is the lowest-order rational solution of the nonlinear Schrödinger (NLS) equation predicted over 30 years ago [8], plays a pivotal role in modeling the extreme waves in different physical branches [9]. It depicts an outlandish doubly localized wave packet featuring a peaked hump three times the significant wave height (SWH) and two side holes that exist as a result of energy conservation. Analytically, it corresponds to the limiting case of either an Akhmediev breather [10] or a Kuznetsov-Ma soliton [11], whenever the oscillating period along the transversal or the propagation direction becomes infinite [2,12]. As the peak position of the Peregrine soliton can be located everywhere, it displays the propensity to appear from nowhere and disappear without a trace [13], which matches the fleeting nature of oceanic rogue waves frequently

\footnotetext{
*cshua@seu.edu.cn

†jsoto@io.cfmac.csic.es

${ }^{\ddagger}$ philippe.grelu@u-bourgogne.fr
}

witnessed by seafarers. To date, aside from nonlinear optics, Peregrine solitons have also been observed in hydrodynamics [14] and in plasma physics [15].

As a fundamental rogue-wave profile, the Peregrine soliton is actually omnipresent in different physical systems. Besides the standard NLS equation [8,16,17], it has been theoretically predicted in derivative or extended NLS equations [18-23], in the Hirota equation [24], and even in the infinite NLS equation hierarchy [25]. It also occurs in vector or multicomponent wave systems (e.g., the two- or three-wave resonant equations [26-28] and the Manakov system [29]) and in higherdimensional wave systems [30]. It is evident that, despite the diversity of integrable systems, all predicted Peregrine solitons inherit the characteristic intensity features - a 3-SWH peak amplitude and two side holes laterally located or slightly tilted.

As a matter of fact, there could be more to it. Different from its simplest form given within the NLS framework $[2,8,14]$, in a more complicated system, the Peregrine soliton would take a different fundamental form; it can acquire an extra nonuniform phase distribution that can give rise to an instantaneous frequency shift also localized in both space and time. This interesting property has not been singled out so far. Besides, earlier work showed that the Peregrine soliton can exist in either the self-focusing or the self-defocusing medium as its solution involves a complex denominator and thus can be relieved from singularity [20,23]; however, what is responsible for this phenomenon is still unclear. Therefore, considering the fact that the electromagnetic energy would flow through phase gradients, hence the new physics that may arise, there is a necessity to study in depth the fundamental form of the Peregrine soliton and reveal its phase evolution dynamics among different systems. Of much interest to the soliton community is how to unify the Peregrine solitons of different phase profiles into a compact form and whether they are stable enough to develop despite the onset of modulation instability (MI). No doubt, to address and clarify these problems will be the primary objectives of the present work. 


\section{GAUGE TRANSFORMATION AND GENERIC PEREGRINE SOLITON SOLUTION}

For our studies, we consider the following dimensionless cubic-quintic (CQ) NLS equation:

$$
\begin{aligned}
& i E_{z}+\frac{1}{2} E_{t t}+\sigma|E|^{2} E+i \gamma\left(|E|^{2} E\right)_{t}+i(\mu-2 \gamma)\left(|E|^{2}\right)_{t} E \\
& \quad+\frac{1}{2}(\mu-\gamma)(\mu-2 \gamma)|E|^{4} E=0,
\end{aligned}
$$

where $E(z, t)$ is the complex envelope of an optical pulse, with $z$ and $t$ the distance and retarded time, respectively. The coefficient $\sigma$ denotes the Kerr nonlinearity, $\gamma$ accounts for the pulse self-steepening effect (we assume $\gamma \geqslant 0$ without loss of generality), and $\mu$ relates to the nonlinearity dispersion, which can result in a self-frequency shift if $\mu$ is complex [31], and the quintic nonlinearity often found in highly nonlinear materials such as chalcogenide glasses [32] and organic polymers [33]. Subscripts $z$ and $t$ stand for partial derivatives. In cases of self-focusing, self-defocusing, and zero Kerr nonlinearities, $\sigma$ can be normalized to $1,-1$, and 0 . It is worth noting that, to gain insight into the fundamental Peregrine form, we have excluded the higher-order dispersion terms (beyond the group-velocity dispersion) in Eq. (1), which usually appear in the high NLS equation hierarchy [25]. In optical contexts, the above equation can model the propagation of ultrashort pulses in a single-mode optical fiber [31] or in quadratic nonlinear media considering the group-velocity mismatch [34].

To attain integrability, we let the coefficients responsible for the self-steepening, nonlinearity dispersion, and quintic nonlinearity be related through two independent real parameters $\gamma$ and $\mu$. In this situation, Eq. (1) can pass Painlevé analysis and is completely integrable [35]. It can reduce to a series of well-established integrable equations such as the well-known standard NLS equation $(\mu=\gamma=0)$, the Chen-Lee-Liu (CLL) -type NLS equation $(\mu=\gamma \neq 0)$ [36], the Kaup-Newell (KN) -type NLS equation $(\mu=2 \gamma)$ [37], the Gerdjikov-Ivanov (GI) equation $(\mu=0)$ [38], and the Kundu-Eckhaus (KE) equation $(\gamma=0)[39,40]$. The rogue-wave solutions of the latter equations were extensively explored in recent years [16,18-22]. However, generic rogue-wave solutions of the integrable CQ NLS equation (1) have not been reported yet; if found, they should include the above known solutions as special cases.

At variance with previous studies, we attempt to solve Eq. (1) by using a simple U(1)-gauge transformation [35,39], rather than using the routine Darboux transformation or Hirota bilinear method. In fact, it is easy to verify that, once the solution of the CLL NLS equation

$$
i u_{z}+\frac{1}{2} u_{t t}+\sigma|u|^{2} u+i \gamma|u|^{2} u_{t}=0
$$

is known, the solution of Eq. (1) can be given by

$$
E(z, t)=u(z, t) \exp [i(\gamma-\mu) \psi]
$$

where the real potential $\psi(z, t)$ is defined through

$$
\psi_{t}=|u|^{2}, \quad \psi_{z}=\frac{i}{2}\left(u^{*} u_{t}-u u_{t}^{*}\right)-\frac{\gamma}{2}|u|^{4} .
$$

Hence, with the gauge transformation (3), we only need to solve Eqs. (4) in terms of the known solution $u$.
We note that the initial plane-wave solution that satisfies Eq. (2) can be expressed as

$$
u_{0}(z, t)=a \exp (i k z+i \omega t)
$$

where $k=\eta a^{2}-\frac{1}{2} \omega^{2}$ and $\eta=\sigma-\gamma \omega$. Then, after some algebra, the fundamental rogue-wave solution of Eq. (2) can be cast into a compact form

$$
u=u_{0}\left[1+\frac{2 i(\gamma \theta-\eta z)-1 / a^{2}}{M-i N}\right],
$$

where $u_{0}$ is given by Eq. (5), $\theta=\left(\omega+\gamma a^{2}\right) z-t$, and

$$
\begin{gathered}
M=\left(\eta+\gamma^{2} a^{2}\right)\left(\theta^{2}+\eta a^{2} z^{2}\right)+\frac{1}{4 a^{2}}, \\
N=\gamma\left(\gamma a^{2} z+\theta\right) .
\end{gathered}
$$

We should point out that our rogue-wave solution (6) generalizes the solution (12) presented in Ref. [20], the latter being obtained under an extra initial condition that can be lifted indeed. Additionally, as its denominator involves an imaginary part $N$, this solution also generalizes the usual Peregrine soliton solution of the NLS equation whose denominator is always real [8]. As we will show later, this nonvanishing imaginary part can give rise to intriguing phase dynamic features.

After insertion of Eq. (6) into Eqs. (4), we find that the potential $\psi$ can be exactly expressed in terms of $M$ and $N$, viz.,

$$
\psi(z, t)=a^{2}\left[t-\left(\omega+\gamma a^{2} / 2\right) z\right]-\frac{2}{\gamma} \arctan (N / M) .
$$

As such, substituting Eq. (9) into Eq. (3), one can readily obtain the rogue-wave solution of Eq. (1) as

$$
E=E_{0}\left[1+\frac{2 i(\gamma \theta-\eta z)-1 / a^{2}}{M-i N}\right] \exp (i \Phi),
$$

where $E_{0}$ and $\Phi$ are the initial plane-wave solution of Eq. (1) and the nonplanar phase, respectively, and are given by

$$
\begin{gathered}
E_{0}(z, t)=u_{0} \exp \left\{i a^{2}(\gamma-\mu)\left[t-\left(\omega+\gamma a^{2} / 2\right) z\right]\right\}, \\
\Phi(z, t)=2(\mu / \gamma-1) \arctan (N / M) .
\end{gathered}
$$

Actually, with the nonplanar phase form given by Eq. (12), the exponential part in Eq. (10) can take another equivalent form

$$
\exp (i \Phi)=\left(\frac{M+i N}{M-i N}\right)^{\mu / \gamma-1} .
$$

If we define the polynomial part within the square brackets in Eq. (10) as a Peregrine function, obviously this complex function has also an inherent phase, which can be defined by

$$
\Theta=\arctan \left[\frac{2 M(\gamma \theta-\eta z)-N / a^{2}}{M^{2}+N^{2}-2 N(\gamma \theta-\eta z)-M / a^{2}}\right] .
$$

In contrast to the acquired phase $\Phi$, however, this inherent phase $\Theta$ does not depend on the parameter $\mu$ and thus will be the same across different integrable systems for given $\gamma$ and given initial parameters. So is the amplitude $|E|$ of the Peregrine soliton. 


\section{CHIRPED PEREGRINE SOLITON DYNAMICS AND DISCUSSION}

An inspection of Eq. (10) reveals that this generic roguewave solution, which exists only when $\eta=\sigma-\gamma \omega>0$, still exhibits a characteristic Peregrine soliton hallmark, namely, a 3-SWH peak amplitude together with two side holes, apart from having an extra nonplanar phase when $\mu \neq \gamma$. For this reason, we dub the solution (10) the chirped Peregrine soliton, which acquires an extra instantaneous frequency shift given by [41]

$$
\delta \omega=-\frac{\partial \Phi}{\partial t}=\frac{2(\mu-\gamma)\left[M-2 N\left(\eta / \gamma+\gamma a^{2}\right) \theta\right]}{M^{2}+N^{2}} .
$$

It is interesting to note that this chirp $\delta \omega$ is also doubly localized, as a rogue wave is, but on a zero background, markedly different from the chirp of traveling dissipative solitons, which is usually of tanh shape in the transversal dimension $[42,43]$. One may find that the magnitude of the chirp (15), in addition to the self-steepening parameter $\gamma$, is also closely related to the quintic nonlinearity that is proportional to $\mu-\gamma$. The chirp will have a central value of $8(\mu-\gamma) a^{2}$, hence displaying a peak or a dip at the center, depending on whether $\mu>\gamma$ or $<\gamma$. Obviously, this kind of strong localization entails potentially important frequency chirping, for large $\mu-\gamma$ values, in both temporal and spatial domains.

We stress that our solution (10) is of the most generic form that unifies different types of Peregrine solitons. It can thus be reduced to the simpler rogue-wave solution reported for the GI equation [19], for the CLL NLS equation [20], and for the KN NLS equation [21], respectively, if the corresponding conditions $\mu=0, \mu=\gamma$, and $\mu=2 \gamma$ are satisfied. We illustrate in Fig. 1 four types of Peregrine solitons formed in the self-focusing situation, showing that they have the same amplitude structure and the same inherent Peregrine phase $\Theta$, as shown in Figs. 1(a) and 1(b), respectively, but have a different extra nonplanar phase $\Phi$, under otherwise identical initial conditions. To be specific, in the CLL NLS situation [see Fig. 1(d)], the extra phase is zero, while in the GI and KN NLS situations, a nonuniform yet contrary phase distribution appears [see Figs. 1(c) and 1(e)]. For other general CQ NLS situations [see, e.g., Fig. 1(f), where we take $\mu=3 \gamma / 2$ ], the phase distribution varies with the value of $\mu$ but has a similar profile, as revealed in Eq. (12).

Moreover, due to the presence of self-steepening effect [refer to the fourth term $\left(|E|^{2} E\right)_{t}$ in Eq. (1) or the fourth term $|u|^{2} u_{t}$ in Eq. (2), as defined in Ref. [34]], this chirped Peregrine soliton can exist in either the self-focusing (anomalous dispersion) or the self-defocusing (normal dispersion) situation. Here we have put the corresponding dispersion situation in parentheses, which is equivalent to the nonlinearity situation after a change of variables. Indeed, the above conclusion is self-evident because the denominator in Eq. (10) is always complex whenever $\gamma \neq 0$ takes place, thus avoiding the singularity that usually occurs in the defocusing situation. In such a case, even if the Kerr nonlinearity is zero (i.e., $\sigma=0$ ), the Peregrine soliton state remains alive as long as $\eta>0$. In particular, we need to emphasize that for the present CQ NLS scenario, the Peregrine soliton form discovered for the
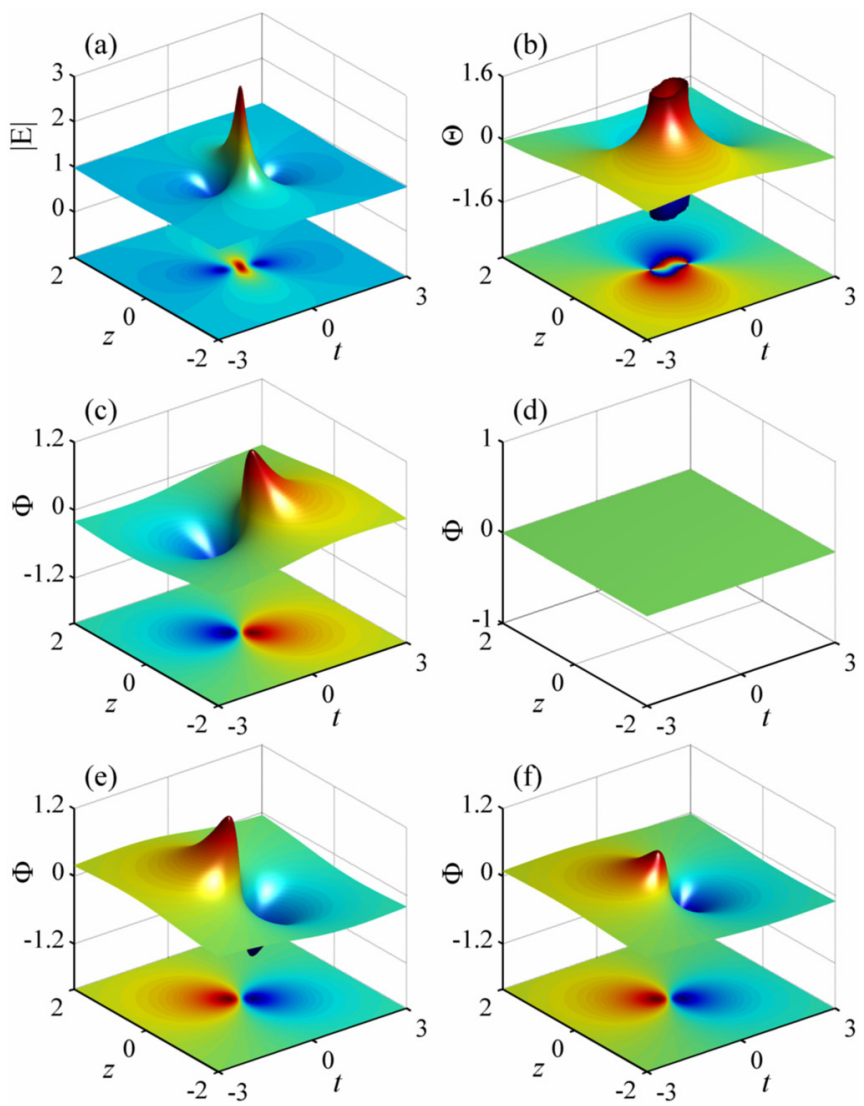

FIG. 1. Three-dimensional and contour plots showing the amplitude $|E|$, which remains the same as in (a), and the nonplanar phase $\Phi$ of chirped Peregrine solitons in the focusing situation $(\sigma=1)$ for given parameters $\gamma=1, a=1$, and $\omega=-1$ : (c) $\mu=0$ (GI equation), (d) $\mu=\gamma$ (CLL NLS equation), (e) $\mu=2 \gamma$ (KN NLS equation), and (f) $\mu=3 \gamma / 2$ (CQ NLS equation). The inherent phase $\Theta$ is illustrated in (b), which remains identical in different $\mu$ cases.

focusing case has no bearing upon the one in the defocusing case, contrarily to the Fokas-Lenells scenario where different types of Peregrine solitons can be linked to each other [23]. Physically, that a defocusing (or, equivalently, normally dispersive) scalar system could allow a bright solution is not surprising. One may recall that in the CQ-type dissipative system, bright dissipative solitons also occur in both the anomalous and normal dispersion regimes [44,45]. Shown in Fig. 2 are the typical Peregrine soliton structure, the total phase, the nonplanar phase, and the corresponding chirp distribution in the case of self-defocusing Kerr nonlinearity, for given parameters $\gamma=1, \mu=1 / 2, a=1$, and $\omega=-2$. It is clear that in such a defocusing situation, a deterministic Peregrine soliton state occurs, usually involving an extra nonplanar phase [see Figs. 2(a)-2(c)]. More intriguingly, the chirp distribution will take a dark doubly localized structure on a zero background for $\mu<\gamma$, as shown in Fig. 2(d).

Now let us take a further look at the Peregrine soliton solution (10) in the limiting case $\gamma \rightarrow 0$ (i.e., in the absence of self-steepening effect). In this case, it follows easily from Eqs. (7) and (8) that

$$
M=\sigma\left[(\omega z-t)^{2}+\sigma a^{2} z^{2}\right]+\frac{1}{4 a^{2}}, \quad N=0
$$



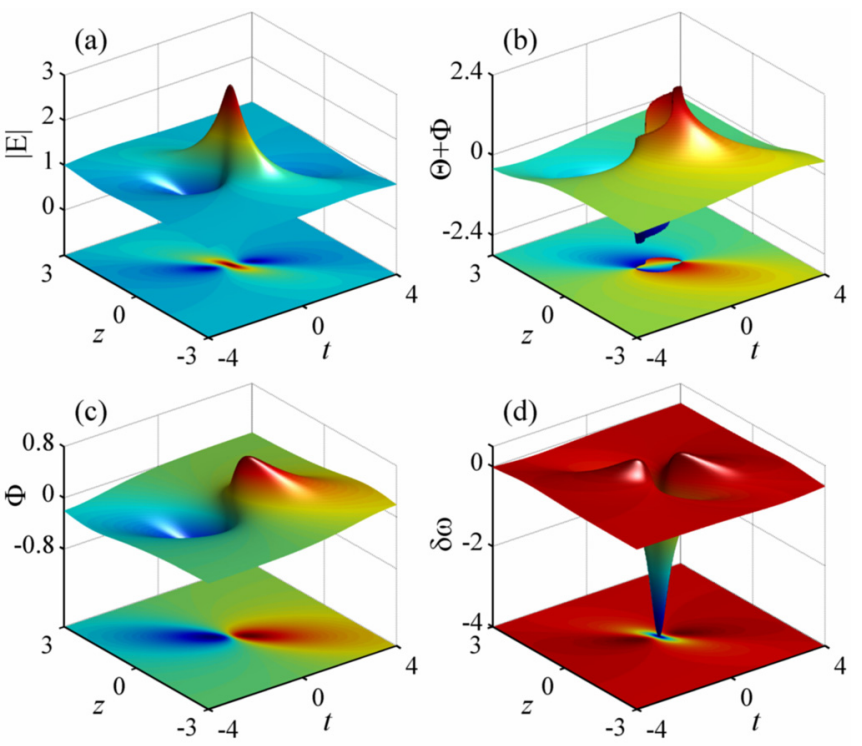

FIG. 2. Generic chirped Peregrine soliton in the self-defocusing situation $(\sigma=-1)$ : (a) the amplitude $|E|$, (b) the total phase $(\Theta+\Phi)$ (excluding the plane-wave phase), (c) the nonplanar phase $\Phi$, and (d) the chirp $\delta \omega$. Other parameters are specified by $\gamma=1, \mu=1 / 2$, $a=1$, and $\omega=-2$.

and from Eq. (12) that $\Phi=2 \mu(\omega z-t) / M$. As a result, Eq. (10) boils down to

$$
E=E_{0}\left(1-\frac{2 i \sigma z+1 / a^{2}}{M}\right) \exp [2 i \mu(\omega z-t) / M]
$$

which is none other than the first-order rational solution of the KE equation [22]. In this case, the chirp formula (15) can also be simplified as $\delta \omega=\frac{2 \mu}{M}\left[1-2 \sigma(\omega z-t)^{2} / M\right]$. With these formulas, one can readily conclude that only in the selffocusing case $(\sigma=1)$ does the KE equation admit rogue-wave solutions, as Eq. (17) involves now a real denominator and would become singular as $\sigma=-1$. Nevertheless, the chirp $\delta \omega$ will take a bright doubly localized structure as $\mu>0$ or a dark one as $\mu<0$. For instance, Fig. 3 shows a typical Peregrine soliton structure along with a bright doubly localized chirp [see Fig. 3(c)] in the focusing KE equation for given $\mu=1 / 2$. In the case of the NLS equation [8], the same amplitude structure as in Fig. 3(a) exists, but the chirp will be absent.
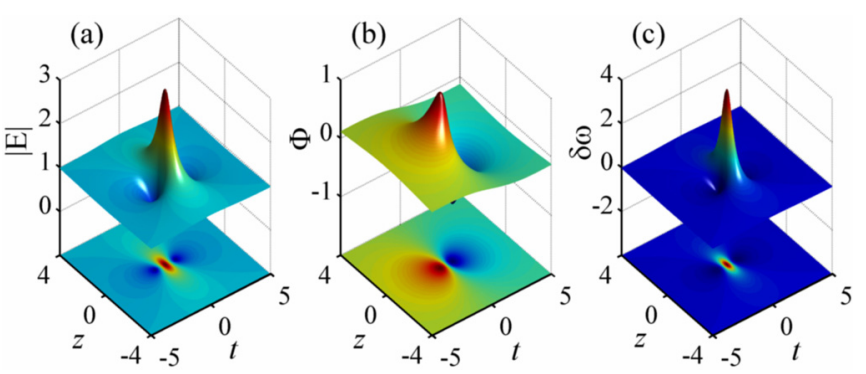

FIG. 3. Chirped Peregrine soliton of the focusing KE equation for given $\sigma=1, \gamma=0, \mu=1 / 2, a=1$, and $\omega=0$ : (a) the amplitude $|E|$, (b) the nonplanar phase $\Phi$, and (c) the chirp $\delta \omega$.
However, this is not always the case with the self-steepening term. Generally, when the higher-order dispersion terms are included in the model, as occurs in the infinite NLS equation hierarchy [25], even under the self-steepening effect, there will be no more Peregrine soliton states for a defocusing Kerr nonlinearity. For instance, if we add a third-order dispersion term $\frac{i \gamma}{6 \sigma} u_{t t t}$ in Eq. (2), it will become the integrable Hirota equation, which admits the following rational solution:

$$
u=u_{0}\left[1-\frac{2 i \eta z+1 / a^{2}}{\sigma\left(\theta-\gamma \omega^{2} z / 2 \sigma\right)^{2}+\eta^{2} a^{2} z^{2}+1 / 4 a^{2}}\right],
$$

where $u_{0}$ is given by Eq. (5) but with $k=\eta a^{2}-\frac{1}{2} \omega^{2}+$ $\frac{\gamma}{6 \sigma} \omega^{3}$. Clearly, different from Eq. (6), which involves an imaginary part $N$, the solution (18) is now endowed with a real denominator and will be singular when $\sigma=-1$, thus precluding the Peregrine soliton state in the defocusing Kerr nonlinearity situation.

\section{MODULATION INSTABILITY AND NUMERICAL SIMULATIONS}

Actually, the general existence condition of the chirped Peregrine soliton $\eta=\sigma-\gamma \omega>0$ can also be obtained via a baseband MI analysis [46]. For this end, we add small-amplitude Fourier modes to the plane-wave solution (11) and express it as $E=E_{0}\{1+p \exp [-i \Omega(\kappa z-t)]+$ $\left.q^{*} \exp \left[i \Omega\left(\kappa^{*} z-t\right)\right]\right\}$, where $p$ and $q$ are small amplitudes of the Fourier modes, $\Omega$ accounts for the modulation frequency $(\Omega \geqslant 0)$, and the propagation parameter $\kappa$ is assumed to be complex. Substituting the above perturbed solution form into Eq. (1) and linearizing the resulting equation, we obtain a system of two coupled linear equations for $p$ and $q$. This system has a nontrivial solution only when $\kappa$ and $\Omega$ satisfy the following dispersion relation:

$$
\left(\kappa-\omega-\gamma a^{2}\right)^{2}+\eta a^{2}-\frac{1}{4} \Omega^{2}=0,
$$

which is a quadratic equation of $\kappa$. In principle, the plane-wave solution (11) becomes unstable whenever $\kappa$ in Eq. (19) has an imaginary part, since the perturbation then grows exponentially with the distance. Obviously, in the limit of $\Omega=0$ (that is, at a sufficiently low modulation frequency in the baseband MI [46]), Eq. (19) admits a pair of complex roots only when $\eta>0$ is satisfied, a sufficient condition exactly consistent with our analytical prediction above.

On the other hand, we define an MI growth rate as $G=$ $\Omega|\operatorname{Im}(\kappa)|$ and find it to be

$$
G=\Omega \sqrt{\eta a^{2}-\Omega^{2} / 4}
$$

which will have a maximum value $\eta a^{2}$ as the modulation frequency equals $\Omega=\sqrt{2 \eta} a$. Figure 4 displays the MI gain map versus $\Omega$ and $\omega$ for both the focusing and defocusing cases. It is clearly shown that the Peregrine soliton states only exist in the $\omega<\sigma / \gamma$ regime for $\gamma \neq 0$, independently of the value of $\mu$, and that for the same initial parameters, the MI in the defocusing case [see Fig. 4(b)] is weaker than in the focusing case [see Fig. 4(a)]. We should point out that in the case of the focusing KE equation, the Peregrine soliton could exist in the whole domain of $\omega$ as the condition $\eta=\sigma>0$ always holds true. Besides, in the present work, 

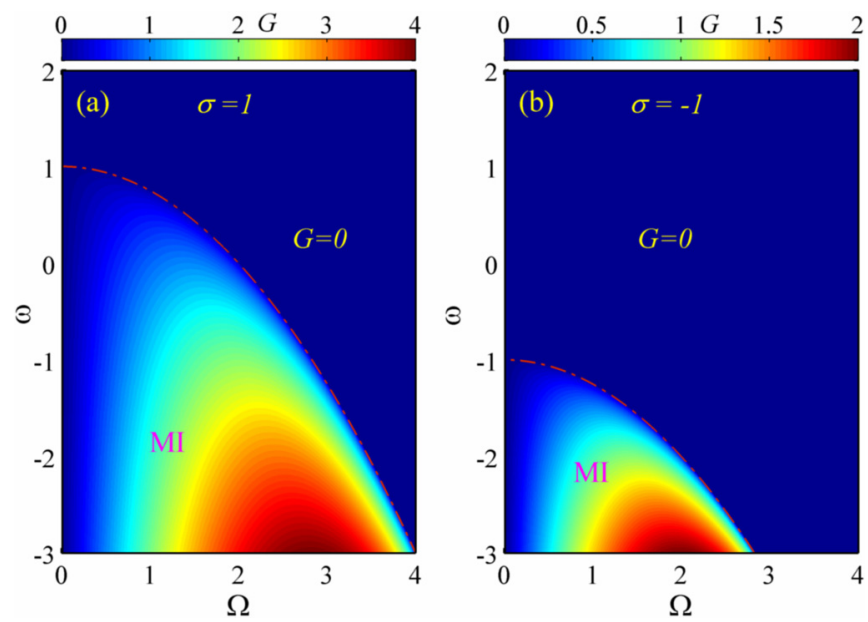

FIG. 4. Map of the MI gain in the plane $(\Omega, \omega)$ for $\gamma=1$ and $a=1$ : (a) $\sigma=1$ (the focusing case) and (b) $\sigma=-1$ (the defocusing case). The red dash-dotted curves stand for the marginal stability defined by $\eta a^{2}-\Omega^{2} / 4=0$.

we are only concerned with the rogue-wave solutions. Indeed, if $\eta \leqslant 0$, Eq. (10) may also serve as a valid solution to Eq. (1), but generally manifests itself in the form of algebraic solitons [23].

We then perform extensive numerical simulations to study the stability of chirped Peregrine solitons in different integrable systems, based on the standard split-step Fourier method [17]. First, we perturb the initial deterministic rogue-wave profile by small amounts of white noise and inspect whether the rogue-wave generation is still observed in the presence of the MI activated by such quantum noise. As usual, we put the noise onto the initial profile by multiplying the real and imaginary parts of the optical field $E$ by a factor $\left[1+\varepsilon r_{i}(x)\right]$ $(i=1,2)$, respectively, where $r_{1,2}$ are two uncorrelated random functions uniformly distributed in the interval $[-1,1]$ and $\varepsilon$ is a small parameter defining the noise level. Figure 5 shows the numerical results of the specific chirped Peregrine soliton given in Fig. 2, which forms with the defocusing Kerr nonlinearity, initially perturbed by white noise of strength $\varepsilon=0.01$. It is exhibited that even under such a large noise perturbation [Fig. 5(a)], this chirped Peregrine soliton, including its amplitude [Fig. 5(b)] and nonplanar phase [Fig. 5(c)], can still propagate very neatly for a rather long distance, with profiles agreeing very well with the analytic solution (10). It is also seen that, once MI is completely developed, the main temporal period of the generated waves is around 4.5 [which will be more obvious in Fig. 6(a) given below], corresponding to a modulation frequency of $2 \pi / 4.5 \approx 1.4$, a value very well consistent with the analytic one $\sqrt{2}$ predicted in our MI analysis above [see Fig. 4(b)].

Second, in order to see whether the chirped Peregrine solitons would be easily generated in realistic conditions, we intend to excite them numerically by using the plane-wave solution (11) at $z=0$ as the initial condition, perturbed by white noise of lower strength $\varepsilon=0.001$. Typical simulation results are presented in Fig. 6, where we used the same plane-wave and model parameters as in Fig. 2 for the sake of comparison. In the amplitude evolution plot [see Fig. 6(a)],
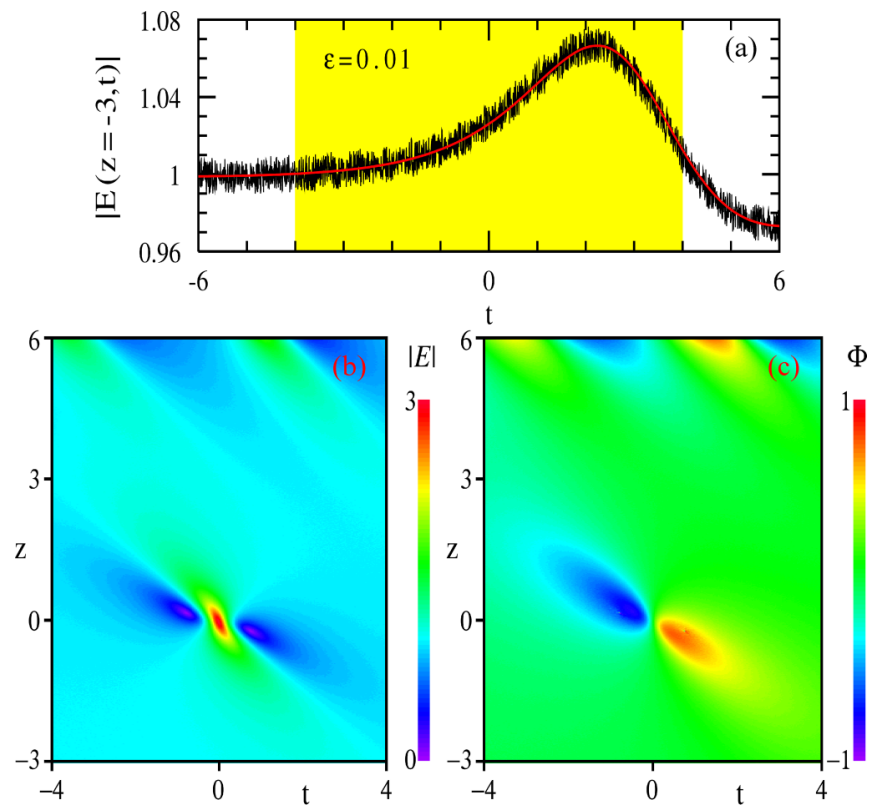

FIG. 5. Simulation results of the chirped Peregrine soliton dynamics shown in Fig. 2 perturbed by white noise of $\epsilon=0.01$ : (a) initial perturbed profile as compared to its analytic solution (red curve), (b) evolution of the amplitude $|E|$, and (c) evolution of the nonplanar phase $\Phi$.

the first five distance units have been removed, as there are hardly any visible changes on the chosen scale. It can be seen that after ten distance units, the MI has developed completely and the isolated rogue waves, the Akhmediev breathers, and the Kuznetsov-Ma solitons in the sea of waves appear. The part selected by a black rectangle is enlarged and plotted in Fig. 6(b). It is clear that the wave in the fore of the 3D plot is exactly the Peregrine soliton shown in Fig. 2, as its profile agrees very well with our analytic solution (10) [see Fig. 6(c)]. This result implies that such chirped Peregrine solitons, for either the focusing or the defocusing Kerr nonlinearity, can be observed in a laboratory environment as long as the model equation (1) applies.

Finally, let us briefly discuss possible experimental settings in the context of nonlinear optics for observation of chirped Peregrine solitons. Usually, one may consider the temporal dynamics of ultrashort pulses in soft glasses such as chalcogenides [32] or in some organic polymers such as polydiacetylene para-toluene sulfonate (PTS) [33], which show observable quintic nonlinearity besides the cubic response. In a bulk PTS crystal, a stable propagation of 2D spatial solitons was reported, due to the stabilizing effect of a saturating quintic nonlinearity [47]. Hence, as one might expect, the generic chirped Peregrine solitons may also be observed in a 1D PTS waveguide, of course taking into account the self-steepening effect that always accompanies the ultrashort pulse propagation [41]. Additionally, one may consider the propagation of ultrashort optical pulses in quadratic crystals (e.g., $\beta$-barium borate crystals, periodically poled lithium niobate, or tantalate crystals $[48,49])$ in the high phase-mismatch cascading regime, which may mimic effective Kerr and selfsteepening effects. We should point out that in this situation, 

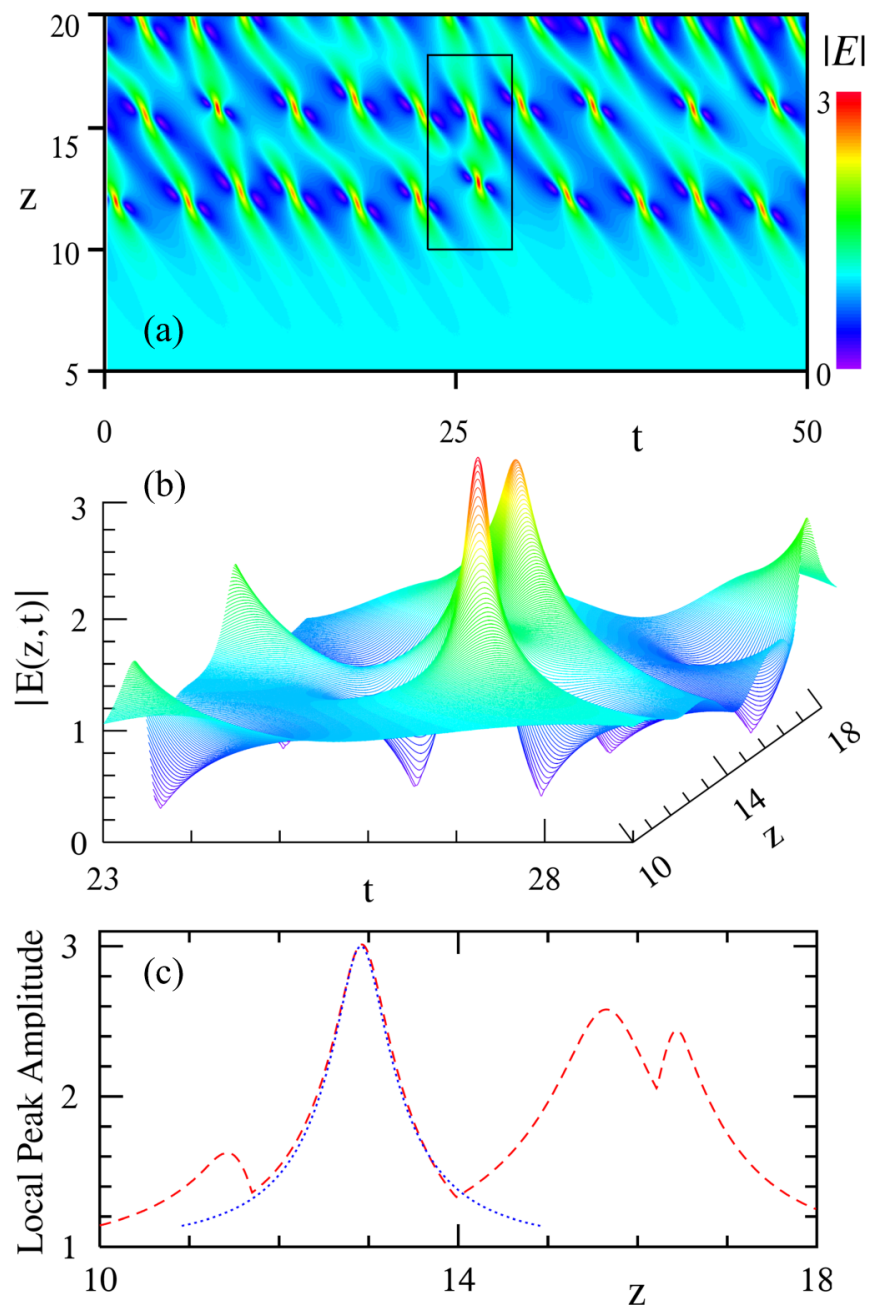

FIG. 6. Numerical excitation of the generic chirped Peregrine soliton state given in Fig. 2 from the plane-wave solution (11) perturbed by white noise of $\epsilon=0.001$ : (a) the amplitude evolution, (b) the enlarged 3D plot of the black rectangle part selected in (a), and (c) the wave profile (red dashed curve) of the selected part fitted by the analytic solution (blue dotted curve).

the propagation of optical pulses can be governed by the CLL NLS equation [48,49], suggesting that our Peregrine solitons shown in Figs. 1(d) and 1(e) may be realized using the same experimental setup. Further, one might also consider modelocked fiber lasers, since their distributed modeling involves quintic nonlinearity [50]. However, the latter is essentially of dissipative type, which breaks down the integrability required by the current analytic approach. Although beyond analyticity, dissipative rogue waves [3] should in general exhibit frequency chirping effects as discussed above. All these interesting issues are topics for future investigation within the soliton physics community.

\section{CONCLUSION}

We obtained an exact generic fundamental rogue-wave solution to the integrable CQ NLS equation via a simple gauge transformation, which unifies the recently-most-studied fundamental rogue-wave species obtained from the standard, derivative, and extended NLS equations [16,18-22]. With insight into its fundamental form and dynamic characteristics, we introduced a more inclusive concept, the chirped Peregrine soliton, for this fundamental rational solution, which generalizes the original Peregrine soliton concept and extends the range of its applicability to cover higher-order nonlinear effects. We demonstrated that this type of Peregrine soliton involves an intriguing doubly localized chirp structure on a zero background, besides having inherited the typical hallmarks such as a 3-SWH peak amplitude and two side holes. This unique chirping property may be attributed to the self-steepening effect or the presence of quintic nonlinearity, or both. In addition, we showed that, aside from the usual focusing nonlinearity situation, the chirped Peregrine soliton can also exist in the self-defocusing nonlinear medium, as long as the self-steepening effect comes into force and is not balanced out by the third-order dispersion. This is contrary to the case in the NLS equation hierarchy where only the unchirped Peregrine soliton is allowed to exist in the selffocusing medium [25]. Finally, extensive simulations were performed to confirm the robustness of chirped Peregrine solitons in both the focusing and defocusing cases, in spite of the onset of the spontaneous MI activated by white noise. We also numerically excited the chirped Peregrine solitons from the background field directly, indicating their observability under realistic experimental conditions. For this purpose, we proposed several feasible experimental schemes as interesting topics for future exploration.

\section{ACKNOWLEDGMENTS}

We thank André Mysyrowicz and Bernard Prade from LOA for helpful discussions. This work was supported by the National Natural Science Foundation of China (Grants No. 11174050 and No. 11474051) and by the Italian Ministry of University and Research (MIUR, Project No. 2012BFNWZ2). P.G. was supported by the Agence Nationale de la Recherche (Project No. ANR-2012-BS04-0011). The work of J.M.S.-C. was supported by MINECO under Contract No. TEC2015-71127-C2-1-R, by CAM under Contract No. S2013/MIT-2790, and by the Volkswagen Foundation.
[1] D. R. Solli, C. Ropers, P. Koonath, and B. Jalali, Optical rogue waves, Nature (London) 450, 1054 (2007).

[2] B. Kibler, J. Fatome, C. Finot, G. Millot, F. Dias, G. Genty, N. Akhmediev, and J. M. Dudley, The Peregrine soliton in nonlinear fibre optics, Nat. Phys. 6, 790 (2010).
[3] C. Lecaplain, P. Grelu, J. M. Soto-Crespo, and N. Akhmediev, Dissipative Rogue Waves Generated by Chaotic Pulse Bunching in a Mode-Locked Laser, Phys. Rev. Lett. 108, 233901 (2012).

[4] M. Leonetti and C. Conti, Observation of three dimensional optical rogue waves through obstacles, Appl. Phys. Lett. 106, 254103 (2015). 
[5] B. Frisquet, B. Kibler, P. Morin, F. Baronio, M. Conforti, G. Millot, and S. Wabnitz, Optical dark rogue wave, Sci. Rep. 6, 20785 (2016).

[6] M. Onorato, S. Residori, U. Bortolozzo, A. Montina, and F. T. Arecchi, Rogue waves and their generating mechanisms in different physical contexts, Phys. Rep. 528, 47 (2013).

[7] J. M. Dudley, F. Dias, M. Erkintalo, and G. Genty, Instabilities, breathers and rogue waves in optics, Nat. Photon. 8, 755 (2014).

[8] D. H. Peregrine, Water waves, nonlinear Schrödinger equations and their solutions, J. Aust. Math. Soc. Ser. B 25, 16 (1983).

[9] V. I. Shrira and V. V. Geogjaev, What makes the Peregrine soliton so special as a prototype of freak waves? J. Eng. Math. 67, 11 (2010).

[10] N. Akhmediev and V. I. Korneev, Modulation instability and periodic solutions of the nonlinear Schrödinger equation, Theor. Math. Phys. 69, 1089 (1986).

[11] E. Kuznetsov, Solitons in a parametrically unstable plasma, Sov. Phys. Dokl. 22, 507 (1977); Y. C. Ma, The perturbed plane-wave solutions of the cubic Schrödinger equation, Stud. Appl. Math. 60, 43 (1979).

[12] B. Kibler, J. Fatome, C. Finot, G. Millot, G. Genty, B. Wetzel, N. Akhmediev, F. Dias, and J. M. Dudley, Observation of Kuznetsov-Ma soliton dynamics in optical fibre, Sci. Rep. 2, 463 (2012).

[13] N. Akhmediev, A. Ankiewicz, and M. Taki, Waves that appear from nowhere and disappear without a trace, Phys. Lett. A 373, 675 (2009).

[14] A. Chabchoub, N. P. Hoffmann, and N. Akhmediev, Rogue Wave Observation in a Water Wave Tank, Phys. Rev. Lett. 106, 204502 (2011)

[15] H. Bailung, S. K. Sharma, and Y. Nakamura, Observation of Peregrine Solitons in a Multicomponent Plasma with Negative Ions, Phys. Rev. Lett. 107, 255005 (2011).

[16] N. Akhmediev, A. Ankiewicz, and J. M. Soto-Crespo, Rogue waves and rational solutions of the nonlinear Schrödinger equation, Phys. Rev. E 80, 026601 (2009).

[17] J. M. Soto-Crespo, N. Devine, and N. Akhmediev, Integrable Turbulence and Rogue Waves: Breathers or Solitons? Phys. Rev. Lett. 116, 103901 (2016).

[18] S. Xu, J. He, and L. Wang, The Darboux transformation of the derivative nonlinear Schrödinger equation, J. Phys. A: Math. Theor. 44, 305203 (2011).

[19] S. Xu and J. He, The rogue wave and breather solution of the Gerdjikov-Ivanov equation, J. Math. Phys. 53, 063507 (2012).

[20] H. N. Chan, K. W. Chow, D. J. Kedziora, R. H. J. Grimshaw, and E. Ding, Rogue wave modes for a derivative nonlinear Schrödinger model, Phys. Rev. E 89, 032914 (2014).

[21] J. He, S. Xu, and Y. Cheng, The rational solutions of the mixed nonlinear Schrödinger equation, AIP Adv. 5, 017105 (2015).

[22] Zhaqilao, On $N$ th-order rogue wave solution to the generalized nonlinear Schrödinger equation, Phys. Lett. A 377, 855 (2013).

[23] S. Chen and L.-Y. Song, Peregrine solitons and algebraic soliton pairs in Kerr media considering space-time correction, Phys. Lett. A 378, 1228 (2014).

[24] A. Ankiewicz, J. M. Soto-Crespo, and N. Akhmediev, Rogue waves and rational solutions of the Hirota equation, Phys. Rev. E 81, 046602 (2010).

[25] A. Ankiewicz, D. J. Kedziora, A. Chowdury, U. Bandelow, and N. Akhmediev, Infinite hierarchy of nonlinear Schrödinger equations and their solutions, Phys. Rev. E 93, 012206 (2016).
[26] S. Chen, P. Grelu, and J. M. Soto-Crespo, Dark- and brightrogue-wave solutions for media with long-wave-short-wave resonance, Phys. Rev. E 89, 011201(R) (2014).

[27] F. Baronio, M. Conforti, A. Degasperis, and S. Lombardo, Rogue Waves Emerging from the Resonant Interaction of Three Waves, Phys. Rev. Lett. 111, 114101 (2013).

[28] S. Chen, F. Baronio, J. M. Soto-Crespo, P. Grelu, M. Conforti, and $\mathrm{S}$. Wabnitz, Optical rogue waves in parametric three-wave mixing and coherent stimulated scattering, Phys. Rev. A 92, 033847 (2015).

[29] F. Baronio, A. Degasperis, M. Conforti, and S. Wabnitz, Solutions of the Vector Nonlinear Schrödinger Equations: Evidence for Deterministic Rogue Waves, Phys. Rev. Lett. 109, 044102 (2012).

[30] A. Kundu, A. Mukherjee, and T. Naskar, Modelling rogue waves through exact dynamical lump soliton controlled by ocean currents, Proc. R. Soc. (London) A 470, 20130576 (2014).

[31] Y. Kodama and A. Hasegawa, Nonlinear pulse propagation in a monomode dielectric guide, IEEE J. Quantum Electron. QE-23, 510 (1987).

[32] G. Boudebs, S. Cherukulappurath, H. Leblond, J. Troles, F. Smektala, and F. Sanchez, Experimental and theoretical study of higher-order nonlinearities in chalcogenide glasses, Opt. Commun. 219, 427 (2003).

[33] B. L. Lawrence, M. Cha, J. U. Kang, W. Toruellas, G. Stegeman, G. Baker, J. Meth, and S. Etemad, Large purely refractive nonlinear index of single crystal P-toluene sulphonate (PTS) at $1600 \mathrm{~nm}$, Electron. Lett. 30, 447 (1994).

[34] J. Moses and F. W. Wise, Controllable Self-Steepening of Ultrashort Pulses in Quadratic Nonlinear Media, Phys. Rev. Lett. 97, 073903 (2006).

[35] P. A. Clarksont and C. M. Cosgrove, Painlevé analysis of the nonlinear Schrödinger family of equations, J. Phys. A: Math. Gen. 20, 2003 (1987).

[36] H. H. Chen, Y. C. Lee, and C. S. Liu, Integrability of nonlinear Hamiltonian systems by inverse scattering method, Phys. Scr. 20, 490 (1979).

[37] D. J. Kaup and A. C. Newell, An exact solution for a derivative nonlinear Schrödinger equation, J. Math. Phys. 19, 798 (1978).

[38] V. S. Gerdjikov and M. I. Ivanov, The quadratic bundle of general form and the nonlinear evolution equations. II. Hierarchies of Hamiltonian structures, Bulg. J. Phys. 10, 130 (1983).

[39] A. Kundu, Landau-Lifshitz and higher-order nonlinear systems gauge generated from nonlinear Schrödinger type equations, J. Math. Phys. 25, 3433 (1984).

[40] W. Eckhaus, The long-time behavior for perturbed waveequations and related problems, Mathematical Institute at University of Utrecht Report No. 404, 1985, Lecture Notes in Physics, Vol. 249 (Springer, Berlin, 1986), pp. 168-194.

[41] G. P. Agrawal, Nonlinear Fiber Optics, 4th ed. (Academic Press, San Diego, 2007)

[42] Z. Li, L. Li, H. Tian, G. Zhou, and K. H. Spatschek, Chirped Femtosecond Solitonlike Laser Pulse form With Self-Frequency Shift, Phys. Rev. Lett. 89, 263901 (2002).

[43] S. Chen and L. Yi, Chirped self-similar solutions of a generalized nonlinear Schrödinger equation model, Phys. Rev. E 71, 016606 (2005).

[44] J. M. Soto-Crespo, N. N. Akhmediev, V. V. Afanasjev, and S. Wabnitz, Pulse solutions of the cubic-quintic complex 
Ginzburg-Landau equation in the case of normal dispersion, Phys. Rev. E 55, 4783 (1997).

[45] S. Chen, Y. Liu, and A. Mysyrowicz, Unusual stability of a one-parameter family of dissipative solitons due to spectral filtering and nonlinearity saturation, Phys. Rev. A 81, 061806(R) (2010).

[46] F. Baronio, S. Chen, P. Grelu, S. Wabnitz, and M. Conforti, Baseband modulation instability as the origin of rogue waves, Phys. Rev. A 91, 033804 (2015).

[47] B. L. Lawrence and G. I. Stegeman, Two-dimensional bright spatial solitons stable over limited intensities and ring formation in polydiacetylene para-toluene sulfonate, Opt. Lett. 23, 591 (1998).

[48] F. Baronio, C. De Angelis, M. Marangoni, C. Manzoni, R. Ramponi, and G. Cerullo, Spectral shift of femtosecond pulses in nonlinear quadratic PPSLT crystals, Opt. Express 14, 4774 (2006).

[49] M. Conforti, F. Baronio, and S. Trillo, Dispersive shock waves in phase-mismatched second-harmonic generation, Opt. Lett. 37, 1082 (2012).

[50] Ph. Grelu and N. Akhmediev, Dissipative solitons for modelocked lasers, Nat. Photon. 6, 84 (2012). 